\title{
Concise Large-Scale Synthesis of Psilocin and Psilocybin, Principal Hallucinogenic Constituents of "Magic Mushroom"
}

\author{
Osamu Shirota, ${ }^{*}{ }^{\dagger}$ Wataru Hakamata, ${ }^{\ddagger}$ and Yukihiro Goda ${ }^{\dagger}$ \\ Division of Pharmacognosy, Phytochemistry and Narcotics, and Division of Organic Chemistry, National Institute of \\ Health Sciences, 1-18-1 Kamiyoga, Setagaya-ku, Tokyo 158-8501, J apan
}

Received February 12, 2003

The concise large-scale syntheses of psilocin (1) and psilocybin (2), the principal hallucinogenic constituents of "magic mushroom", were achieved without chromatographic purification. The key step in the synthesis of $\mathbf{2}$ was the isolation of the dibenzyl-protected intermediate (7) as a zwitterionic derivative (8), which was completely identified by means of 2D NMR analyses.

\begin{abstract}
"Magic mushrooms"1 is the name most commonly given to hallucinogenic fungi containing the psychoactive constituents psilocin (1) and psilocybin (2), 2,3 the principal active constituents of Psilocybemushrooms. Baeocystin and norbaeocystin are often minor constituents. ${ }^{4,5}$ These compounds closely resemble the neurotransmitter serotonin, and the hallucinogenic effect of the "magic mushroom" is probably caused by their interference with the normal actions of brain seretonin. ${ }^{6,7}$ It is likely that LSD works in a similar fashion. ${ }^{8}$ The use of "magic mushrooms" has become popular among young people because it is relatively inexpensive, and there is lower awareness of guilt than with other drugs. ${ }^{9-11}$ Therefore, since J une 6, 2002, fungi containing $\mathbf{1}$ and $\mathbf{2}$ have been regulated by the Narcotics and Psychotropic Control Law in J apan. The identification of the "magic mushroom" using morphologic and microscopic analyses is quite difficult without experts, so that chromatographic methods including TLC, GC, and HPLC are usually employed. ${ }^{12-17}$ For these chromatographic analyses, standard compounds are always needed. It is difficult to isolate $\mathbf{1}$ and $\mathbf{2}$ from the mushroom on a gram scale for use as pure standard compounds because 1 easily decomposes and $\mathbf{2}$ has a high polarity. Several reports on the synthesis of 1 have been published, ${ }^{18-25}$ while reports on the synthesis of $\mathbf{2}$ are few. ${ }^{18,19,24}$ We report herein concise large-scale syntheses of psilocin (1) and psilocybin (2) that were achieved without any chromatographic purification.

The syntheses, summarized in Scheme 1, started from commercially available 4-hydroxyindole (3) with simple protection of the hydroxyl group by acetylation. Similar protection by benzyl ether was also utilized; 24 however, a separate step was needed for its deprotection. In the next step, $\mathbf{4}$ afforded $\mathbf{5}$ as yellow crystals by treatment with oxalyl chloride, whereas the 4-O-benzyl derivative of $\mathbf{3}$ was somewhat unstable, without careful control of the reaction conditions, and was not isolated in crystal form. Thus, 4 was subjected to a two-step acylation-amidation conversion to obtain the glyoxalylamide (6) in over $80 \%$ yield. Reduction of $\mathbf{6}$ by $\mathrm{LiAlH}_{4}$ then afforded psilocin (1) in over $85 \%$ yield.
\end{abstract}

F or the synthesis of $\mathbf{2}$, the phosphorylated derivative of 1, several phosphorylation methodologies were applied. Most of the phosphorylation methods did not consume 1; however, the phosphoryl iodide method, ${ }^{26}$ using tribenzyl

* To whom correspondence should be addressed. Tel/Fax: +81-3-37009165. E-mail: shirota@nihs.go.jp.

† Division of Pharmacognosy, Phytochemistry and Narcotics.

₹ Division of Organic Chemistry. phosphite, $I_{2}$, and DMAP, and the pyrophosphate method, ${ }^{24,27}$ using tetrabenzylpyrophosphate and n-BuLi, appeared promising. Because of its easy handling and the reagent stability, the pyrophosphate method was selected to produce $\mathbf{7}$ on a large scale. In this reaction, $\mathbf{1}$ was smoothly consumed and a newly formed spot was then principally observed on TLC. After the usual aqueous workup for removing the excess reagents, the ${ }^{1} \mathrm{H}$ NMR spectrum of the remaining substance in $\mathrm{CDCl}_{3}$ showed complicated signals. Rechecking the TLC showed an additional spot at the origin, and the whitish material no longer dissolved in $\mathrm{CH}_{2} \mathrm{Cl}_{2}$. A similar observation has been reported by Nichols and Frescas, who concluded that hydrolytic cleavage of one of the O-benzyl groups rapidly occurred and the resulting zwitterionic O-monobenzyl phosphate was obtained as a mixture. ${ }^{24}$ Our purification effort by preparative reversed-phase HPLC afforded a single compound (8), which was analyzed again by NMR. The ${ }^{1} \mathrm{H}$ and ${ }^{13} \mathrm{C}$ NMR spectra of 8 in $\mathrm{CD}_{3} \mathrm{OD}$ showed signals for two sets of benzyl groups and a psilocin core, although the proton signal of the methylene on one benzyl group was shifted to high field $\left(\delta_{H} 4.56,2 \mathrm{H}, \mathrm{s}\right)$ compared to the signals of the other $\left(\delta_{\mathrm{H}} 4.98,1 \mathrm{H}, \mathrm{s} ; 4.96,1 \mathrm{H}, \mathrm{s}\right)$, while the proton signals of the two sets of methylene and $\mathrm{N}, \mathrm{N}$-dimethyl parts on the psilocin core were shifted to low field compared to those of psilocin itself. The 31P NMR spectrum confirmed the presence of the phosphate moiety in the molecule. These data suggested the intramolecular conversion of the benzyl-bearing sites on $\mathbf{7}$. Confirmation of this assumption was achieved using an HMBC experiment. The HMBC spectrum revealed that one benzyl group was directly linked at the nitrogen of the $\mathrm{N}, \mathrm{N}$-dimethyl part (a quaternary ammonium ion). The NOESY spectrum al so supported these linkages. The observed key correlations are illustrated in Figure 1. These data suggested that $\mathbf{8}$ was a zwitterionic N,O-dibenzyl phosphate derivative. The conversion of the 0,0-dibenzyl phosphate derivative (7) into this zwitterionic N,O-di benzyl phosphate derivative (8) was easily achieved by suspending the worked-up reaction mixture in $\mathrm{CH}_{2} \mathrm{Cl}_{2}$ overnight. The zwitterionic nature of $\mathbf{8}$ made possible its large-scale isolation by filtration, in over $85 \%$ yield, since the excess remaining dibenzyl phosphate was removed by washing with $\mathrm{CH}_{2} \mathrm{Cl}_{2}$. Catalytic hydrogenolysis of $\mathbf{8}$ then led to psilocybin (2) as a crystalline product without any chromatographic purification such as the anion-exchange resin that was used by Nichols and Frescas. ${ }^{24}$ The isolated yield of $\mathbf{2}$ from $\mathbf{1}$ was greater than $72 \%$, even for a gram-scale production, and was quite 
Scheme $1^{\text {a }}$
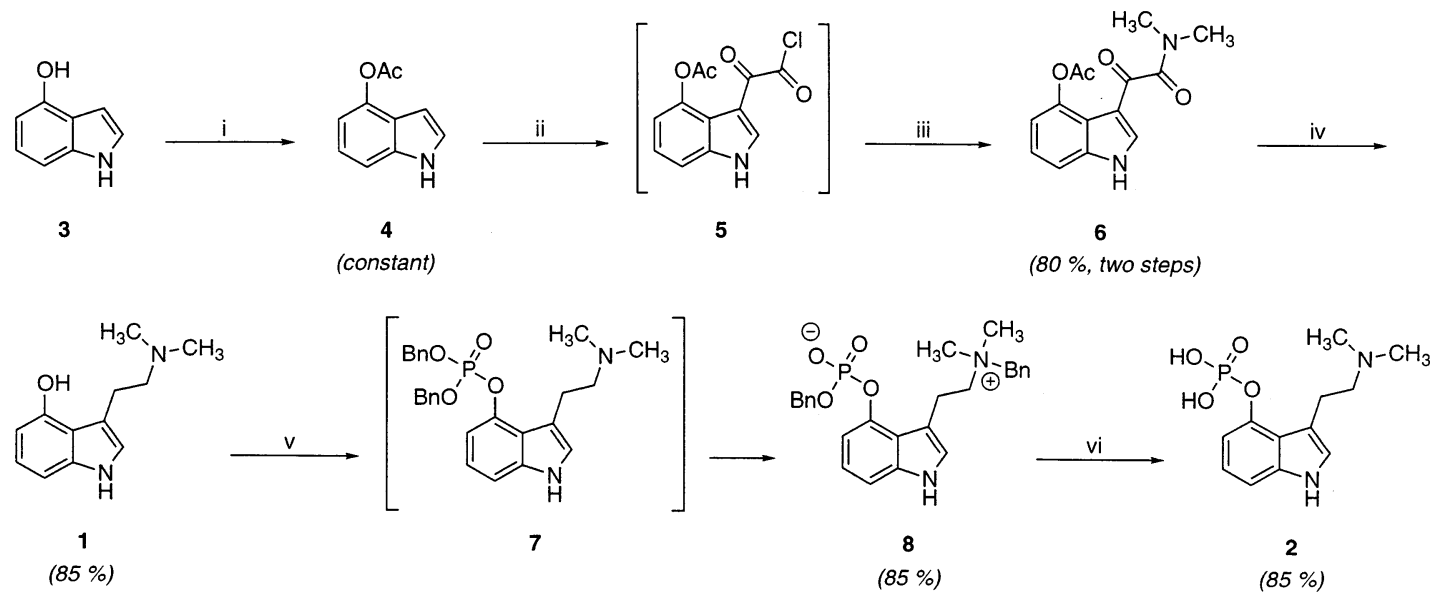

a Reagent and conditions: (i) $\mathrm{Ac}_{2} \mathrm{O}$, pyridine, $\mathrm{CH}_{2} \mathrm{Cl}_{2}, 0{ }^{\circ} \mathrm{C}$ to rt; (ii) $(\mathrm{COCl})_{2}$, ether, $0{ }^{\circ} \mathrm{C}$, n-hexane, then $-20{ }^{\circ} \mathrm{C}$; (iii) $\left(\mathrm{CH}_{3}\right)_{2} \mathrm{NH}, \mathrm{THF}^{\circ}$; (iv) $\mathrm{LiAlH}$, $\mathrm{THF}$, $\Delta$; (v) $\left[(\mathrm{BnO})_{2} \mathrm{PO}\right]_{2} \mathrm{O}, \mathrm{n}-\mathrm{BuLi}, \mathrm{THF},-78{ }^{\circ} \mathrm{C}$ to $0{ }^{\circ} \mathrm{C}$; (vi) $\mathrm{H}_{2}, \mathrm{Pd} / \mathrm{C}, \mathrm{MeOH}, \mathrm{rt}$.

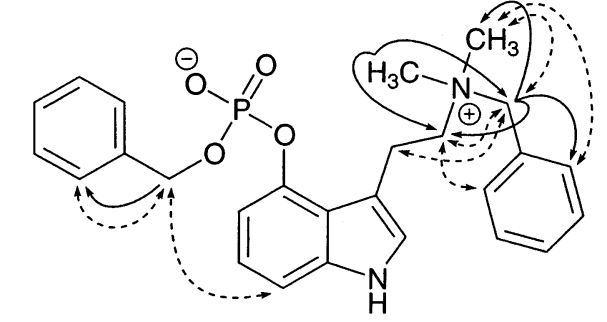

: key HMBC correlations W: key NOESY correlations

Figure 1. Key HMBC and NOESY correlations of 8.

gratifying when compared to previously reported yields of $20 \% 18,19$ and $47 \% .^{24}$

In conclusion, gram scale syntheses of the principal hallucinogenic constituents in "magic mushrooms", psilocin (1) and psilocybin (2), were readily achieved, with no chromatographic purification needed. The latter compound was prepared via a newly identified zwitterionic N,Odibenzyl phosphate intermediate (8), which was fully identified by means of 2D NMR analyses.

\section{Experimental Section}

General Experimental Procedures. Commercial reagents were used without purification. TLC was performed on precoated silica gel $60 \mathrm{~F}_{254}$ (Merck) or aminopropyl silica gel (Chromatorex NH; Fuji Silysia Chemical, Ltd., Aichi, J apan), and spots were visualized by heating with Ehrlich's reagent and/or by UV light at $254 \mathrm{~nm}$. Melting points were determined on a Yanagimoto micromelting point apparatus and were uncorrected. The UV and IR spectra were recorded on a Shimadzu UV-2550 spectrophotometer and a J ASCO FT/IR5300 spectrophotometer, respectively. The ESIMS and ESIHRMS spectra were obtained using API QSTAR Pulsar i and/ or J EOL AccuTOF spectrometers. The one and two-dimensional NMR spectra were recorded on Varian spectrometers (Mercury 400 and Unity 400 plus) at ambient temperature using standard pulse sequences. TMS was used as the internal standard for the ${ }^{1} \mathrm{H}$ and ${ }^{13} \mathrm{C} N \mathrm{NMR}$, and phosphoric acid was used as the external standard for ${ }^{31}$ P NMR. For measurement of psilocybin (2), a solvent residue peak (HDO) was used for the ${ }^{1} \mathrm{H}$ NMR reference, and one drop of $\mathrm{MeOH}$ was added as the reference of the ${ }^{13} \mathrm{C}$ NMR. Chemical shifts are reported in $\delta$, and coupling constants $(\mathrm{J})$ are given in $\mathrm{Hz}$.

4-Acetylindole (4). To a solution of 4-hydroxyindole (3; Tokyo Kasei Kogyo Co., Ltd.; > 25 g/bottle, > 185 mmol) in anhydrous $\mathrm{CH}_{2} \mathrm{Cl}_{2}(200 \mathrm{~mL}$ ) with stirring in an ice bath was added pyridine (20 mL, $246 \mathrm{mmol}$ ) and acetic anhydride (20
$\mathrm{mL}, 210 \mathrm{mmol}$ ). After the mixture was stirred for $2 \mathrm{~h}$ at room temperature, $\mathrm{H}_{2} \mathrm{O}$ was added, and the mixture was evaporated in vacuo. The resulting concentrate was dissolved in ethyl acetate and washed twice with $\mathrm{H}_{2} \mathrm{O}$ and once with saturated $\mathrm{NaCl}$. The organic phase was dried over anhydrous $\mathrm{Na}_{2} \mathrm{SO}_{4}$ and the volume reduced by evaporation to form a crystalline material, which was collected by filtration and successively washed with $\mathrm{H}_{2} \mathrm{O}$ and ethyl acetate to afford 4 (34 g; constant) as ivory white crystals: ${ }^{1} \mathrm{H} \mathrm{NMR}\left(\mathrm{CDCl}_{3}, 400 \mathrm{MHz}\right) \delta 8.27(1 \mathrm{H}$, br s, H-1), $7.22(1 \mathrm{H}, \mathrm{d}, \mathrm{J}=8.0 \mathrm{~Hz}, \mathrm{H}-7), 7.15(1 \mathrm{H}, \mathrm{t}, \mathrm{J}=8.0$ $\mathrm{Hz}, \mathrm{H}-6), 7.11(\mathrm{H}, \mathrm{t}, \mathrm{J}=2.8 \mathrm{~Hz}, \mathrm{H}-2), 6.85(1 \mathrm{H}, \mathrm{dd}, \mathrm{J}=0.5$, $8.0 \mathrm{~Hz}, \mathrm{H}-5), 6.41(1 \mathrm{H}, \mathrm{m}, \mathrm{H}-3), 2.39\left(3 \mathrm{H}, \mathrm{s}, \mathrm{OCOCH}_{3}\right) ;{ }^{13} \mathrm{C}$ $\mathrm{NMR}\left(\mathrm{CDCl}_{3}, 100 \mathrm{MHz}\right) \delta 169.6\left(\mathrm{C}, \mathrm{OCOCH}_{3}\right), 143.6(\mathrm{C}, \mathrm{C}-4)$, $137.6(\mathrm{C}, \mathrm{C}-7 \mathrm{a}), 124.5(\mathrm{CH}, \mathrm{C}-2), 122.1(\mathrm{CH}, \mathrm{C}-6), 121.2(\mathrm{C}$, C-3a), $111.8(\mathrm{CH}, \mathrm{C}-5), 109.2(\mathrm{CH}, \mathrm{C}-7), 99.2(\mathrm{CH}, \mathrm{C}-3), 21.1$ $\left(\mathrm{CH}_{3}, \mathrm{OCOCH}_{3}\right) ; \mathrm{ESIMS} \mathrm{m} / \mathrm{z} 198.0[\mathrm{M}+\mathrm{Na}]^{+}(63), 176.1$ $[\mathrm{M}+\mathrm{H}]^{+}$(53), $134.0[\mathrm{M}-\mathrm{Ac}+\mathrm{H}]^{+}$(100). This material was directly used in the next step.

3-Dimethylaminooxalyl-4-acetylindole (6). To a solution of $4(17.6 \mathrm{~g}, 100 \mathrm{mmol})$ in anhydrous diethyl ether $(100 \mathrm{~mL})$ with stirring in an ice bath was added oxalyl chloride $(13 \mathrm{~mL}$, $146 \mathrm{mmol})$. After stirring for $15 \mathrm{~min}$, n-hexane $(200 \mathrm{~mL}$ ) was added, and the reaction flask was placed in a freezer and stored overnight. The resulting yellow crystal (5) was separated from the solution by filtration and dissolved in anhydrous tetrahydrofuran (100 mL). Tothis solution with stirring in an ice bath was added a $2 \mathrm{M}$ dimethylamine tetrahydrofuran solution $(60 \mathrm{~mL}, 120 \mathrm{mmol})$ and pyridine $(10 \mathrm{~mL}, 123$ $\mathrm{mmol}$ ) over $15 \mathrm{~min}$. Additional anhydrous ether was added to the mixture because of solidification, and then it was stirred for $15 \mathrm{~min}$ at room temperature. The reaction product was separated from the solution by filtration and successively washed with n-hexane, ethyl acetate, and $\mathrm{H}_{2} \mathrm{O}$ to afford $\mathbf{6}$ (22.0 $\mathrm{g}, 80.0 \%)$ as an ivory white crystalline powder: ${ }^{1} \mathrm{H}$ NMR $\left(\mathrm{CDCl}_{3}, 400 \mathrm{MHz}\right) \delta 10.40(1 \mathrm{H}, \mathrm{br} \mathrm{s}, \mathrm{H}-1), 7.52(1 \mathrm{H}, \mathrm{d}, \mathrm{J}=3.2$ $\mathrm{Hz}, \mathrm{H}-2), 7.15(1 \mathrm{H}, \mathrm{t}, \mathrm{J}=8.0 \mathrm{~Hz}, \mathrm{H}-6), 7.06(1 \mathrm{H}, \mathrm{d}, \mathrm{J}=8.0$ $\mathrm{Hz}, \mathrm{H}-7), 6.91(1 \mathrm{H}, \mathrm{d}, \mathrm{J}=8.0 \mathrm{~Hz}, \mathrm{H}-5), 3.02\left(3 \mathrm{H}, \mathrm{s}, \mathrm{NCH}_{3}\right)$, $2.92\left(3 \mathrm{H}, \mathrm{s}, \mathrm{NCH}_{3}\right), 2.50\left(3 \mathrm{H}, \mathrm{s}, \mathrm{OCOCH}_{3}\right) ;{ }^{13} \mathrm{C} \mathrm{NMR}\left(\mathrm{CDCl}_{3}\right.$, $100 \mathrm{MHz}) \delta 185.4\left(\mathrm{C}, \mathrm{C}-1^{\prime}\right), 170.9\left(\mathrm{C}, \mathrm{OCOCH}_{3}\right), 168.4\left(\mathrm{C}, \mathrm{C}-2^{\prime}\right)$, 144.2 (C, C-4), 139.2 (C, C-7a), 138.2 (CH, C-2), 124.7 (CH C-6), 118.2 (C, C-3a), 116.0 (CH, C-5), 113.5 (C, C-3), 110.8 $(\mathrm{CH}, \mathrm{C}-7), 37.4\left(\mathrm{CH}_{3}, \mathrm{NCH}_{3}\right), 34.2\left(\mathrm{CH}_{3}, \mathrm{NCH}_{3}\right), 21.6\left(\mathrm{CH}_{3}\right.$, $\left.\mathrm{OCOCH}_{3}\right) ; \mathrm{ESIMS} \mathrm{m} / \mathrm{z} 297.1\left[\mathrm{M}+\mathrm{Na}^{+}(77), 275.1\left[\mathrm{M}+\mathrm{H}^{+}\right.\right.$ (77), $233.1[\mathrm{M}-\mathrm{Ac}+\mathrm{H}]^{+}$(100). This material was directly used in the next step.

Psilocin (1). To a suspension of lithium aluminum hydride (ca. $12 \mathrm{~g}$ ) in anhydrous tetrahydofuran $(300 \mathrm{~mL})$ under an argon atmosphere was dropwise added a solution of $\mathbf{6}(22.0 \mathrm{~g}$, $80 \mathrm{mmol})$ in anhydrous tetrahydofuran $(250 \mathrm{~mL})$ over $2 \mathrm{~h}$, and then the reaction mixture was refluxed for $2 \mathrm{~h}$. After cooling, anhydrous $\mathrm{Na}_{2} \mathrm{SO}_{4}$ powder (ca. $10 \mathrm{~g}$ ) was added, and then a solution of saturated $\mathrm{Na}_{2} \mathrm{SO}_{4}$ (ca. $12 \mathrm{~mL}$ ) was dropwise added 
over $1 \mathrm{~h}$ with stirring at room temperature. After the reaction was stopped, additional anhydrous $\mathrm{Na}_{2} \mathrm{SO}_{4}$ powder (ca. $10 \mathrm{~g}$ ) was added. The reaction mixture was then diluted with ethyl acetate and filtered through an aminopropyl silica gel laminated Celite pad by suction. The pad was washed with ethyl acetate. The organic solution was quickly concentrated in vacuo, and the resulting crystals were briefly washed with $\mathrm{MeOH}$ to afford psilocin (1; $14.3 \mathrm{~g}, 87.5 \%)$ as white crystals: mp 169-174 dec ${ }^{\circ} \mathrm{C}$ (lit. ${ }^{3} \mathrm{mp} 173-176 \mathrm{dec}{ }^{\circ} \mathrm{C}$ ); UV (MeOH) $\lambda_{\max }(\log \epsilon) 222.5$ (4.55), 268.0 (3.72), 284.5 (3.62), 294.0 (3.58) $\mathrm{nm}$; IR (KBr) $v_{\max } 3285,2959,2371,1620,1588,1473,1345$, $1258,1232,1044,833,722 \mathrm{~cm}^{-1}$; ${ }^{1} \mathrm{H} \mathrm{NMR}\left(\mathrm{CDCl}_{3}, 400 \mathrm{MHz}\right)$ $\delta 7.90(1 \mathrm{H}, \mathrm{br} \mathrm{s}, \mathrm{H}-1), 7.05(1 \mathrm{H}, \mathrm{d}, \mathrm{J}=8.0 \mathrm{~Hz}, \mathrm{H}-6), 6.86(1 \mathrm{H}$, $\mathrm{dd}, \mathrm{J}=0.8,8.0 \mathrm{~Hz}, \mathrm{H}-7), 6.84(1 \mathrm{H}, \mathrm{d}, \mathrm{J}=2.4 \mathrm{~Hz}, \mathrm{H}-2), 6.56$ $(1 \mathrm{H}, \mathrm{dd}, \mathrm{J}=0.8,8.0 \mathrm{~Hz}, \mathrm{H}-5), 2.94\left(2 \mathrm{H}, \mathrm{m}, \mathrm{H}_{2}-\mathrm{I}^{\prime}\right), 2.70(2 \mathrm{H}$, $\left.\mathrm{m}, \mathrm{H}_{2}-2^{\prime}\right), 2.38\left(6 \mathrm{H}, \mathrm{s}, \mathrm{NMe}_{2}\right) ;{ }^{13} \mathrm{C} \mathrm{NMR}\left(\mathrm{CDCl}_{3}, 100 \mathrm{MHz}\right) \delta$ 152.1 (C, C-4), 139.0 (C, C-7a), 123.5 (CH, C-6), $120.8(\mathrm{CH}$, C-2), 117.5 (C, C-3a), 114.6 (C, C-3), 106.4 (CH, C-5), 102.4 $(\mathrm{CH}, \mathrm{C}-7), 61.6\left(\mathrm{CH}_{2}, \mathrm{C}-2^{\prime}\right), 45.3\left(\mathrm{CH}_{3} \times 2, \mathrm{NMe}_{2}\right), 25.1\left(\mathrm{CH}_{2}\right.$, C-1'); ESIMS m/z $227.1[\mathrm{M}+\mathrm{Na}]^{+}$(42), $205.1[\mathrm{M}+\mathrm{H}]^{+}(100)$, $160.1\left[\mathrm{M}-\mathrm{NMe}_{2}\right]^{+}(96) ; \mathrm{HRESIMS} \mathrm{m/z} 205.1303[\mathrm{M}+\mathrm{H}]^{+}$ (calcd for $\mathrm{C}_{12} \mathrm{H}_{17} \mathrm{~N}_{2} \mathrm{O}, 205.1341$ ).

\{Benzyl[2-(4-oxyindol-3-yl)ethyl]dimethylammonio\}4-0-benzyl Phosphate (8). To a solution of $\mathbf{1}$ (5.4 g, 26.4 $\mathrm{mmol})$ in anhydrous tetrahydofuran $(265 \mathrm{~mL})$ with stirring at $-78^{\circ} \mathrm{C}$ was added $2.6 \mathrm{M}$ n-butyllithium in $\mathrm{n}$-hexane $(11.5 \mathrm{~mL}$, $29.9 \mathrm{mmol}$ ). After stirring for $5 \mathrm{~min}$, tetrabenzyl pyrophosphate $(18.0 \mathrm{~g}, 33.4 \mathrm{mmol})$, which was prepared in almost $100 \%$ yield from dibenzyl phosphate using a literature procedure with some modification, ${ }^{27}$ was added all at once to the mixture. Stirring was continued for $1 \mathrm{~h}$ while the temperature was allowed to warm to $0^{\circ} \mathrm{C}$. After checking the production of $\mathbf{7}$, instead of the disappearance of $\mathbf{1}$, aminopropyl silica gel (ca. $20 \mathrm{~g}$ ) was added to the reaction mixture, and then the mixture was diluted with ethyl acetate and filtered through a Celite pad by suction. The filtrate was concentrated in vacuo, redissolved in $\mathrm{CH}_{2} \mathrm{Cl}_{2}$, and stored overnight. The precipitated white substance was collected by filtration and washed with $\mathrm{CH}_{2} \mathrm{Cl}_{2}$ to obtain $\mathbf{8}(10.5 \mathrm{~g}, 85.2 \%)$ as a white powder: ${ }^{1} \mathrm{H} \mathrm{NMR}$ $\left(\mathrm{CD}_{3} \mathrm{OD}, 400 \mathrm{MHz}\right) \delta 7.56-7.45\left(5 \mathrm{H}, \mathrm{m}, \mathrm{NCH}_{2} \mathrm{C}_{6} \mathrm{H}_{5}\right), 7.31-$ $7.20\left(5 \mathrm{H}, \mathrm{m}, \mathrm{OCH}_{2} \mathrm{C}_{6} \mathrm{H}_{5}\right), 7.12(1 \mathrm{H}, \mathrm{d}, \mathrm{J}=7.8 \mathrm{~Hz}, \mathrm{H}-7), 7.10$ $(1 \mathrm{H}, \mathrm{br}, \mathrm{H}-2), 7.09(1 \mathrm{H}, \mathrm{d}, \mathrm{J}=7.8 \mathrm{~Hz}, \mathrm{H}-5), 7.01(1 \mathrm{H}, \mathrm{t}, \mathrm{J}=$ $7.8 \mathrm{~Hz}, \mathrm{H}-6), 4.98,4.96$ (each $\left.\mathrm{IH}, \mathrm{s}, \mathrm{OCH}_{2} \mathrm{C}_{6} \mathrm{H}_{5}\right), 4.56(2 \mathrm{H}, \mathrm{s}$, $\left.\mathrm{NCH}_{2} \mathrm{C}_{6} \mathrm{H}_{5}\right), 3.64\left(2 \mathrm{H}, \mathrm{m}, \mathrm{H}_{2}-2^{\prime}\right), 3.47\left(2 \mathrm{H}, \mathrm{m}, \mathrm{H}_{2}-\mathrm{I}^{\prime}\right), 3.08(6 \mathrm{H}$, $\left.\mathrm{s}, \mathrm{NMe}_{2}\right) ;{ }^{13} \mathrm{C} \mathrm{NMR}\left(\mathrm{CD}_{3} \mathrm{OD}, 100 \mathrm{MHz}\right) \delta 147.7$ (C, split, C-4), 140.5 (C, C-7a), $139.3\left(\mathrm{C}, \mathrm{C}_{5} / \mathrm{OCH}_{2} \mathrm{C}_{6} \mathrm{H}_{5}\right), 134.2(\mathrm{CH} \times 2$, $\left.\mathrm{C} / \mathrm{NCH}_{2} \mathrm{C}_{6} \mathrm{H}_{5}\right), 131.8\left(\mathrm{CH}, \mathrm{C}_{\mathrm{p}} / \mathrm{NCH}_{2} \mathrm{C}_{6} \mathrm{H}_{5}\right), 130.2(\mathrm{CH} \times 2$, $\left.\mathrm{C}_{\mathrm{m}} / \mathrm{NCH}_{2} \mathrm{C}_{6} \mathrm{H}_{5}\right), 129.3\left(\mathrm{CH} \times 2, \mathrm{C}_{m} / \mathrm{OCH}_{2} \mathrm{C}_{6} \mathrm{H}_{5}\right), 129.1$ (C, $\left.\mathrm{C}_{5} / \mathrm{NCH}_{2} \mathrm{C}_{6} \mathrm{H}_{5}\right), 128.8\left(\mathrm{CH} \times 2, \mathrm{C}_{0} / \mathrm{OCH}_{2} \mathrm{C}_{6} \mathrm{H}_{5}\right), 128.7(\mathrm{CH}$, $\left.\mathrm{C}_{\mathrm{p}} / \mathrm{OCH}_{2} \mathrm{C}_{6} \mathrm{H}_{5}\right), 124.4(\mathrm{CH}, \mathrm{C}-2), 123.3(\mathrm{CH}, \mathrm{C}-6), 120.2(\mathrm{C}$, split, C-3a), 110.1 (CH, C-7), 109.0 (C, C-3), 108.2 (CH, C-5), $69.2\left(\mathrm{CH}_{2}, \mathrm{NCH}_{2} \mathrm{C}_{6} \mathrm{H}_{5}\right), 69.1\left(\mathrm{CH}_{2}\right.$, split, $\left.\mathrm{OCH}_{2} \mathrm{C}_{6} \mathrm{H}_{5}\right), 67.6\left(\mathrm{CH}_{2}\right.$, $\left.\mathrm{C}-2^{\prime}\right), 50.3\left(\mathrm{CH}_{3} \times 2, \mathrm{NMe}_{2}\right), 21.5\left(\mathrm{CH}_{2}, \mathrm{C}-\mathrm{I}^{\prime}\right) ;{ }^{31} \mathrm{P} \mathrm{NMR}\left(\mathrm{CD}_{3}-\right.$ $\mathrm{OD}, 162 \mathrm{MHz}) \delta-5.45\left(\mathrm{P}, \mathrm{OPO}_{3} \mathrm{CH}_{2} \mathrm{C}_{6} \mathrm{H}_{5}\right) ; \mathrm{ESIMS} \mathrm{m} / \mathrm{z} 487.2$ $[\mathrm{M}+\mathrm{Na}]^{+}(54), 465.2[\mathrm{M}+\mathrm{H}]^{+}(100), 385.2(31), 295.2[\mathrm{M}-$ $\left.\mathrm{C}_{7} \mathrm{H}_{7} \mathrm{O}_{3} \mathrm{P}+\mathrm{H}\right]^{+}(51), 160.1\left[\mathrm{M}-\mathrm{C}_{7} \mathrm{H}_{7} \mathrm{O}_{3} \mathrm{P}-\mathrm{NMe}_{2}\right]^{+}(51) ;$ HRESIMS m/z 465.1883 [M + H] $]^{+}$(calcd for $\mathrm{C}_{26} \mathrm{H}_{30} \mathrm{~N}_{2} \mathrm{O}_{4} \mathrm{P}$, 465.1943). This material was used directly in the next step.

Psilocybin (2). To a solution of 8 (10.5 g, $22.5 \mathrm{mmol})$ in $\mathrm{MeOH}(225 \mathrm{~mL})$ was added $10 \%$ palladium-activated carbon (ca. $1 \mathrm{~g}$ ) under an argon atmosphere, and the suspension was stirred under a hydrogen atmosphere at room temperature. Two hours later, $\mathrm{H}_{2} \mathrm{O}$ (ca. $50 \mathrm{~mL}$ ) was added to the mixture because of product deposition, and the mixture was stirred for one more hour under a hydrogen atmosphere. After the disappearance of $\mathbf{8}$ and its mono debenzyl derivative, and the appearance of $\mathbf{2}$ (on TLC), the reaction solution was filtered through a Celite pad by suction, and the volume was reduced by evaporation to form crystalline material. The product was collected by filtration and washed with $\mathrm{EtOH}$ to afford psilocybin (2; $5.6 \mathrm{~g}, 87.5 \%)$ as a white needle crystalline powder: $\mathrm{mp} 190-198{ }^{\circ} \mathrm{C}$ (lit. $2,28 \mathrm{mp} 185-195{ }^{\circ} \mathrm{C}, 210-212{ }^{\circ} \mathrm{C}$ ); UV $(\mathrm{MeOH}) \lambda_{\max }(\log \epsilon) 221.0$ (4.44), 267.5 (3.66), 278.5 (3.57), 290.0 (3.42) nm; IR (KBr) $v_{\max } 3266,3034,2731,2369,1620,1580$, $1505,1439,1352,1298,1244,1154,1103,1061,926,858,804$ $\mathrm{cm}^{-1} ;{ }^{1} \mathrm{H}$ NMR $\left(\mathrm{D}_{2} \mathrm{O}, 400 \mathrm{MHz}\right) \delta 7.22(1 \mathrm{H}, \mathrm{d}, \mathrm{J}=7.6 \mathrm{~Hz}, \mathrm{H}-7)$, $7.18(1 \mathrm{H}, \mathrm{s}, \mathrm{H}-2), 7.13(1 \mathrm{H}, \mathrm{t}, \mathrm{J}=7.6 \mathrm{~Hz}, \mathrm{H}-6), 6.98(1 \mathrm{H}, \mathrm{d}$, $\mathrm{J}=7.6 \mathrm{~Hz}, \mathrm{H}-5), 3.44\left(2 \mathrm{H}, \mathrm{t}, \mathrm{J}=7.2 \mathrm{~Hz}, \mathrm{H}_{2}-2^{\prime}\right), 3.28(2 \mathrm{H}, \mathrm{t}$, $\left.\mathrm{J}=7.2 \mathrm{~Hz}, \mathrm{H}_{2}-\mathrm{I}^{\prime}\right), 2.86\left(6 \mathrm{H}, \mathrm{s}, \mathrm{NMe}_{2}\right) ;{ }^{13} \mathrm{C} \mathrm{NMR}\left(\mathrm{D}_{2} \mathrm{O}+1 \mathrm{drop}\right.$ of $\mathrm{MeOH}, 100 \mathrm{MHz}) \delta 146.4$ (C, split, C-4), 139.4 (C, C-7a), 124.8 (CH, C-6), 123.3 (CH, C-2), 119.1 (C, split, C-3a), 109.5 $\left(\mathrm{CH}\right.$, split, C-5a), 108.6 (C, C-3), $108.4(\mathrm{CH}, \mathrm{C}-7), 59.7\left(\mathrm{CH}_{2}\right.$ $\left.\mathrm{C}-2^{\prime}\right), 43.4\left(\mathrm{CH}_{3} \times 2, \mathrm{NMe}_{2}\right), 22.4\left(\mathrm{CH}_{2}, \mathrm{C}-1^{\prime}\right) ;{ }^{31} \mathrm{P} \mathrm{NMR}\left(\mathrm{CD}_{3-}\right.$ $\mathrm{OD}, 162 \mathrm{MHz}) \delta-4.48\left(\mathrm{P}, \mathrm{OPO}_{3} \mathrm{H}_{2}\right) ; \mathrm{ESIMS} \mathrm{m} / \mathrm{z} 307.1[\mathrm{M}+$ $\mathrm{Na}]^{+}$(53), $285.1[\mathrm{M}+\mathrm{H}]^{+}$(100), $240.0\left[\mathrm{M}-\mathrm{NMe}_{2}\right]^{+}$(16), 205.1 $\left[\mathrm{M}-\mathrm{H}_{2} \mathrm{O}_{3} \mathrm{P}+\mathrm{H}\right]^{+}(26), 160.1\left[\mathrm{M}-\mathrm{H}_{2} \mathrm{O}_{3} \mathrm{P}-\mathrm{NMe}_{2}\right]^{+}(12) ;$ HRESIMS m/z $285.0991[\mathrm{M}+\mathrm{H}]^{+}$(calcd for $\mathrm{C}_{12} \mathrm{H}_{18} \mathrm{~N}_{2} \mathrm{O}_{4} \mathrm{P}$, 285.1004)

Acknowledgment. This work was supported by a research grant from the Ministry of Health, Labour and Welfare of J apan.

\section{References and Notes}

(1) Wasson, R. G. Life 1957, 42, 100-120.

(2) Hofmann, A.; Heim, R.; Kobel, H. Experientia 1958, 14, 107-109.

(3) Hofmann, A.; Heim, R.; Brack, A.; Kobel, H.; Frey, A.; Ott, H.; Petrzilka, T.; Troxler, F. Helv. Chim. Acta 1959, 42, 1557-1572.

(4) Leung, A. Y.: Paul, A. G. J. Pharm. Sci. 1967, 56, 146.

(5) Leung, A. Y.; Paul, A. G. J. Pharm. Sci. 1968, 57, 1667-1671.

(6) Strassman, R. J. Neuropsychopharmacology 1992, 7, 241-243.

(7) Vollenweider, F. X.; Vollenweider-Scherpenhuyzen, M. F.; Babler, A.; Vogel, H.; Hell, D. Neuroreport 1998, 9, 3897-3902.

(8) McCall, R. B. Neurosci. Biobehav. Rev. 1982, 6, 509-514.

(9) Adlaf, E. M.; Paglia, A.; I vis, F. J .; Ialomiteanu, A. Cmaj 2000, 162, $1677-1680$

(10) Lohrer, F.; Kaiser, R. Nervenarzt 1999, 70, 1029-1033.

(11) Westberg, U.; Karlson-Stiber, C. Lakartidningen 1999, 96, 746-747.

(12) Bogusz, M. J. . . Chromatogr. B Biomed. Sci. Appl. 2000, 748, 3-19.

(13) Keller, T. Schneider A.; Regenscheit, P. Dirnhofer, R.; Rucker, T. J aspers, J .; Kisser, W. Forensic Sci. Int. 1999, 99, 93-105.

(14) Marcano, V.; Morales-Mendez, A.; Castellano, F.; Salazar, F. J .; Martinez, L. J Ethnopharmacol. 1994, 43, 157-159.

(15) Kysilka, R.; Wurst, M.J. Chromatogr. 1989, 464, 434-437.

(16) Merlin, M. D.; Allen, J. W. J . Ethnopharmacol. 1993, 40, 21-40.

(17) Beug, M. W.; Bigwood, J. J . Chromatogr. 1981, 207, 379-385.

(18) Troxler, F.; Seemann, F.; Hofmann, A. Helv. Chim. Acta 1959, 42, 2073-2103.

(19) Hofmann, A.; Frey, A.; Ott, H.; Petrzilka, T.; Troxler, F. Experientia 1958, 15, 397-399.

(20) Yamada, F.; Tamura, M.; Somei, M. Heterocycles 1998, 49, 451.

(21) Ono, M.; Shimamine, M.; Takahashi, K. Bull. Nat. Inst. Hygienic Sci. 1973, 91, 39-41.

(22) Repke, D. B.; Ferguson, W. J .; Bates, D. K. J . Heterocycl. Chem. 1981 $18,175-179$.

(23) Ametamey, S.; Vollenweider, F. X.; Patt, J .; Bourquin, D.; Hasler F.; Beer, H.-F .; Schubiger, P. A. J . Label. Compd. Radiopharm. 1998 $41,585-594$.

(24) Nichols, D. E.; Frescas, S. Synthesis 1999, 935-938.

(25) Sakagami, H.: Ogasawara, K. Heterocycles 1999, 51, 1131-1135.

(26) Stowell, J. K.; Widlanski, T. S. Tetrahedron Lett. 1995, 36, 18251826.

(27) Almeida, M. V. d.; Dubreuil, D.; Cleophax, J .; Verre-Sebrié, C.; Pipelier, M.; Prestat, G.; Vass, G.; Gero, S. D. Tetrahedron 1999, 55, $7251-7270$.

(28) The Combined Chemical Dictionary on CD-ROM; Chapman \& Hall/ CRC, 2002

NP030059U 